\title{
History and Future of Automated Vulnerability Analysis
}

\author{
Adam Doupé \\ doupe@asu.edu \\ Arizona State University
}

\begin{abstract}
The software upon which our modern society operates is riddled with security vulnerabilities. These vulnerabilities allow hackers access to our sensitive data and make our system insecure. To identify vulnerabilities in software, human experts, or vulnerability researchers, are employed. These human experts are quite expensive. And, more fundamentally, human experts cannot analyze every change made to every piece of software (any of which could introduce a security vulnerability). Therefore, automated vulnerability analysis techniques were developed to automatically perform the process of identifying security vulnerabilities in software systems. These tools attempt to democratize the vulnerability analysis process: allowing any developer to identify vulnerabilities in their software automatically, thus finding such vulnerabilities before a malicious hacker.

In this keynote, I will discuss the history of automated vulnerability analysis, from both the binary and the web perspective. Binary fuzzing and black-box web application vulnerability analysis have many aspects in common, yet are often thought of separately. From this, I will discuss the future of automated vulnerability analysis, and how we can achieve the effectiveness of a human vulnerability researcher.
\end{abstract}

\section{CCS CONCEPTS}

- Security and privacy $\rightarrow$ Vulnerability scanners; Web application security.

\section{KEYWORDS}

vulnerability analysis; automated vulnerability analysis; fuzzing; web security; binary security

\section{ACM Reference Format:}

Adam Doupé. 2019. History and Future of Automated Vulnerability Analysis. In The 25th ACM Symposium on Access Control Models and Technologies (SACMAT '19), June 3-6, 2019, Toronto, ON, Canada. ACM, New York, NY, USA, 1 page. https://doi.org/10.1145/3322431.3326331
Permission to make digital or hard copies of part or all of this work for personal or classroom use is granted without fee provided that copies are not made or distributed for profit or commercial advantage and that copies bear this notice and the full citation on the first page. Copyrights for third-party components of this work must be honored

For all other uses, contact the owner/author(s).

SACMAT '19, June 3-6, 2019, Toronto, ON, Canada

(C) 2019 Copyright held by the owner/author(s)

ACM ISBN 978-1-4503-6753-0/19/06.

https://doi.org/10.1145/3322431.3326331

\section{BIOGRAPHY}

Dr. Adam Doupé is an Assistant Professor in the School of Computing, Informatics, and Decision Systems Engineering at Arizona State University and is the Associate Director of the Center for Cybersecurity and Digital Forensics in the Global Security Initiate at Arizona State University. Dr. Doupé was awarded the Top 5\% Faculty Teaching Award for the Fulton Schools of Engineering at ASU in 2016 and 2019, the Fulton Schools of Engineering Best Teacher Award in 2017 and 2018, the Fulton Schools of Engineering Outstanding Assistant Professor Award in 2017, and the NSF CAREER award in 2017. Dr. Doupé has co-authored over 20 peer-reviewed scholarly publications and served on program committees of wellknown international security conferences. As a founding member of the Order of the Overflow, Dr. Doupe has organized the DEF CON Capture The Flag competition since 2018.

\section{ACKNOWLEDGMENTS}

This material is based upon work supported by the National Science Foundation under Grant No. 1651661. 